\title{
Multiresolution analysis on nonlinear complexity measurement of EEG signal for epileptic discharge monitoring
}

\author{
Mohd Syakir Fathillah a, ${ }^{\star}$, Rosmina Jaafar ${ }^{a}$, Kalaivani Chellappan ${ }^{a}$, Rabani Remli b, Wan Asyraf \\ Wan Zainal ${ }^{b}$ \\ a Department of Electrical, Electronic and System, Faculty of Engineering and Built Environment, Univesiti Kebangsaan Malaysia, 43600 Bangi, \\ Selangor, Malaysia \\ ${ }^{b}$ Neurology Unit, Department of Medicine, Pusat Perubatan Universiti Kebangsaan Malaysia, Cheras Malaysia \\ * Corresponding author: syakirfathillah92@gmail.com
}

\section{Article history}

Submitted 15 October 2017

Received 6 February 2018

Accepted 20 April 2018

Published Online 4 June 2018

\begin{abstract}
Various epileptic discharge detection studies have been conducted however, not many clinically significant outcomes have been achieved in developing reliable algorithm using nonlinear measurement techniques. Study has reported that some of the linear measurement techniques performs better than nonlinear technique in detecting the epileptic discharge in terms of accuracy, sensitivity and specificity. The reliability issue has been addressed in nonlinear techniques, by introducing multiresolution analysis (MRA). MRA with approximate entropy are the most common combination used to detect epileptic discharge, leaving other nonlinear complexity measures are yet to be explored with MRA. Previously, we have study the performance of Approximate Entropy (ApEn) and Lempel Ziv (LZ) using MRA. In this paper, we have expanded the scope by studying performance of MRA with other complexity measurement including Hurst exponent (HE), Kolmogorov complexity (KC), Shannon Entropy (SE) and Sample Entropy (SampEn). Groups of normal with interictal (Set A and $B$ ), normal with ictal (Set $A$ and $C$ ) and interictal with ictal (Set $B$ and $C$ ) were used to evaluate the performance. For the result, MRA managed to enhance the accuracy of ApEn (AB: $74 \%$ to $89 \%$, AC: $98 \%$ to $100 \%$, BC: $88 \%$ to $94 \%$ ) and SE (AB: $69 \%$ to $98 \%$, AC: $100 \%$ to $100 \%$, BC: $96 \%$ to $97 \%$ ) in detecting epileptic discharge the best while deteriorating LZ (AB: $49 \%$ to $83 \%$, AC: $91 \%$ to $86 \%$, $\mathrm{BC}: 89 \%$ to $88 \%$ ) and $\mathrm{HE}$ (AB: $65 \%$ to $70 \%$, $\mathrm{AC}: 89 \%$ to $78 \%$, BC: $80 \%$ to $54 \%$ ) performance. Computation time tends to increase with the implementation of MRA.
\end{abstract}

Keywords: Multiresolution analysis, nonlinear complexity measurement, electroencephalograph, seizure

(C) 2018 Penerbit UTM Press. All rights reserved

\section{INTRODUCTION}

The Electroencephalogram (EEG) is non-stationary signal which condition to produce this signal vary over time, making EEG signal to exhibit nonstationary, nonlinear, stochastic, dynamic and also complex behaviour (Klonowski, 2009). These properties have been used by previous researcher to investigate in EEG study related to characterization of brain function (Grassberger et al., 1991), study of emotional (Li et al., 2016), cognitive processing (Natarajan et al. 2004), measurement of depth of anaesthesia (Zhang et al., 2001), and seizure detection (Ocak, 2009).

Dimensionless nature of nonlinear technique is an advantage in predicting and detecting epileptic event (Alotaiby et al., 2014; Blanco et al., 2013; Clemson et al., 2016). The same technique is applicable in localization and characterisation of epileptic region (Stam, 2005), which could assist in automated epileptic discharge detection (Gajic et al., 2015).

Detecting epileptic discharge is important for a neurologist to diagnose epilepsy. Conventional method by visual screening are tedious and time consuming activity. Development of epileptic discharge detection algorithim is the solution for this limitation. Nonlinear complexity measurement is suitable to be applied as epileptic discharge detection algorithm due to the release of excessive and synchronous of this discharge (Fisher et al., 2005). The presence of epileptic discharge in EEG signal will lower the complexity of the signal (Smith, 2005), thus making it a suitable feature to be extracted to differentiate normal and epileptic EEG. Despite numerous of studies were conducted in this area, limited progress has been achieved so far (Nagaraj et al., 2015). Study has reported that some of linear measurement techniques performances are better than nonlinear technique in detecting the epileptic discharge (Nagaraj et al., 2015).

Past researchers have combined multiresolution analysis (MRA) with nonlinear analysis in epileptic discharge study to enhance the performance. Implementation of MRA with Lyapunov exponent and Correlation dimension demonstrated good performance in discriminating seizure with non-seizure (Gajic et al., 2015). In (Ocak, 2009), MRA is implemented along with approximate entropy. Another utilization of MRA with approximate entropy are reported in (Kumar et al., 2014; Wang et al., 2017). Based on literature finding, most algorithm implement approximate entropy as complexity measurement with MRA. Other complexity measurement techniques such as Lempel Ziv, Hurst exponent, Kolmogorov complexity and Shannon entropy are yet to be explored. 
The core concern of this research is to identify the best potential complexity measurement algorithm to be applied with MRA approach in detecting epileptic discharge. The outcome of this research is to provide a suggestion for potential technique to be used in clinical environment and aid in reducing the neurologist diagnosing time for epilepsy. In our previous study we focused on effect of MRA on Lempel Ziv and ApEn (Fathillah et al., 2017). In this paper, the study has been expanded by involving additional complexity measurement technique such as Hurst exponent, Kolmogorov complexity, Shannon entropy and Sample entropy. Performance measurement in detecting epileptic discharge between with and without implementation of MRA were observed. The automated epileptic discharge detection was developed using support vector machine (SVM) to classify between normal, interictal and ictal epileptic discharge.

\section{METHODOLOGY}

In this study, EEG data were processed using MRA. Complexity features are extracted from each sub-band using nonlinear complexity measurements and the outcome were classified using SVM. The work flow is shown as in Fig. 1.

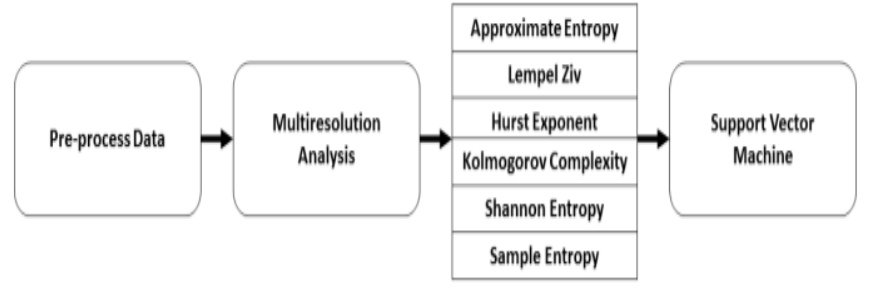

Fig. 1 Workflow of proposed methodology.

\section{Data Source}

This study utilizes three sets of online public EEG data (A, B, and C) which were acquired from the Department of Epileptology, University of Bonn database (Andrzejak et al., 2012). Each set contains 100 single channels that were recorded using 128-channel amplifier system. The duration of each data is 23.6 seconds, sampled at $173.61 \mathrm{~Hz}$ and band-pass filtered from $0.53 \mathrm{~Hz}$ to $40 \mathrm{~Hz}$. Set A consists of 5 healthy subjects where the subjects were awake and relaxed with eye opened. For set B, it contains interictal epileptic discharge (interictal seizure) and was recorded within epileptogenic region. Set $\mathrm{C}$ contained ictal epileptic discharge (ictal seizure) activities. The interictal epileptic discharge has same characteristic as ictal, but in shorter duration and no symptom shown by the patient (Staley \& Dudek, 2006). The electrodes placement is according to international 10-20 system. The summarization of datasets detail is as in Table 1.

Table 1 Summary of clinical data.

\begin{tabular}{|c|c|c|c|}
\hline & Set $1(A)$ & Set 2 (B) & Set $3(C)$ \\
\hline $\begin{array}{l}\text { Subject } \\
\text { Condition }\end{array}$ & $\begin{array}{l}\text { Healthy } \\
\text { subject with } \\
\text { eyes open }\end{array}$ & $\begin{array}{c}\text { Interictal } \\
\text { epileptic } \\
\text { discharge }\end{array}$ & $\begin{array}{l}\text { Ictal epileptic } \\
\text { discharge }\end{array}$ \\
\hline $\begin{array}{l}\text { Electrode } \\
\text { placement }\end{array}$ & $\begin{array}{c}\text { International } \\
10-20 \text { systems }\end{array}$ & $\begin{array}{l}\text { Within } \\
\text { epileptogenic } \\
\text { zone }\end{array}$ & $\begin{array}{l}\text { Within } \\
\text { epileptogenic } \\
\text { zone }\end{array}$ \\
\hline
\end{tabular}

Seizure data is utilized in this study to demonstrate the change of complexity during normal, interictal and seizure period. During the release of epileptic discharge, EEG signal appear regular and repetitive (Ocak 2009) compare to normal EEG. Fig. 2 shows the comparison of EEG signal between normal, interictal and ictal discharge.

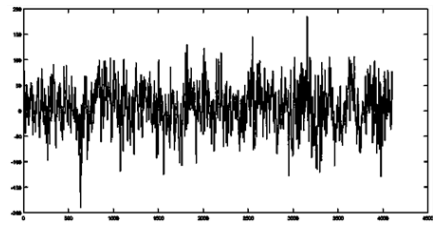

(a)

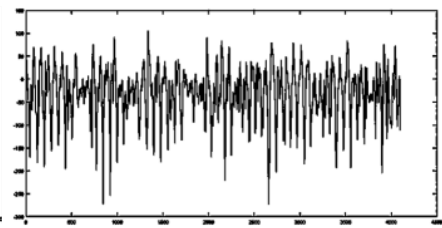

(b)

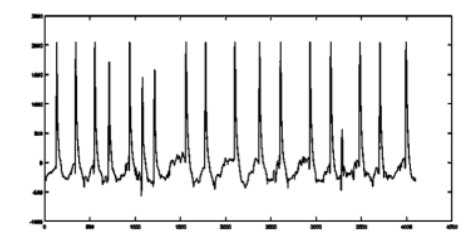

(c)

Fig. 2. Complexity pattern of EEG signal (a) Normal (b) Interictal (c) Ictal.

\section{Signal Processing}

Epileptic EEG have the non-stationary and transient characteristic, in such condition application of frequency domain transformation is inefficient. With the application of time-frequency transformation such as wavelet transform, transient abnormal pattern occurs at various points in the signal can be detected. Wavelet transform is a convenient approach as it can provide precise information regarding frequency when the signal frequency is low and accurate information in time when signal frequency is high. Wavelet transform can be divided into two categories, Continuous Wavelet Transform and Discrete Wavelet Transform. In this study, we will focus on implementation of DWT due to its ability to employ MRA and can be described by the equation (1).

$f(t)=\sum_{j \in Z} 2^{j} / 2 c_{j}(k) \varphi\left(2^{j} t-k\right)+\sum_{j=0}^{j-1} \sum_{k=0}^{\infty} 2^{j / 2} d_{j}(k) \omega\left(2^{j} t-l\right)$

where $\phi(t)$ is a scaling function, $\psi(t)$ is a basis function and $\mathrm{j}$ is the scale index. Initially, the sampling frequency will be divided by two according to Nyquist theorem. The signal then undergoes high pass filter and low pass filter that will produce an approximation of $f(t)$ and detail of $f(t)$ respectively which will be presented in finer scale. The cut-off frequency of high-pass and low-pass filter is equal to one-fourth of the sampling frequency.

The types of wavelets play important role in the wavelet transform. In this study, we adopted Daubechies 4 (db4) as mother wavelet because of its smoothing feature and suitability in detecting EEG changes (Omerhodzic et al., 2010). Db4 also has advantages in orthogonality property and efficient filter implementation (Adeli et al., 2003). Decomposition level is set to five to correlate it with classification of wave.

The result of this process is the sub-band decomposition, which are to be used in this study. The sub-bands are represent by coefficient A5, D5, D4, D3, D2 and D1. Coeffients A5 is product of approximation of $f(t)$ while other coffiencient is detail of $f(t)$. Complexity feature is extracted for all the coeffients using complexity measurement that will be discussed later. Fig. 3 shows decomposition of signal into 6 subbands frequency

Table 2 Coefficient and its representation of frequency band.

\begin{tabular}{cccc}
\hline Coefficient & $\begin{array}{c}\text { Frequency } \\
\text { Band (Hz) }\end{array}$ & Type of wave & $\begin{array}{c}\text { Level of } \\
\text { Decomposition }\end{array}$ \\
\hline D1 & $43.40-86.81$ & Noise & 1 \\
D2 & $21.70-43.40$ & Beta-Gamma & 2 \\
D3 & $10.85-21.70$ & Alpha-Beta & 3 \\
D4 & $5.43-10.85$ & Theta-Alpha & 4 \\
D5 & $2.71-5.43$ & Delta-Theta & 5 \\
A5 & $0.5-2.71$ & Delta & 5 \\
\hline
\end{tabular}




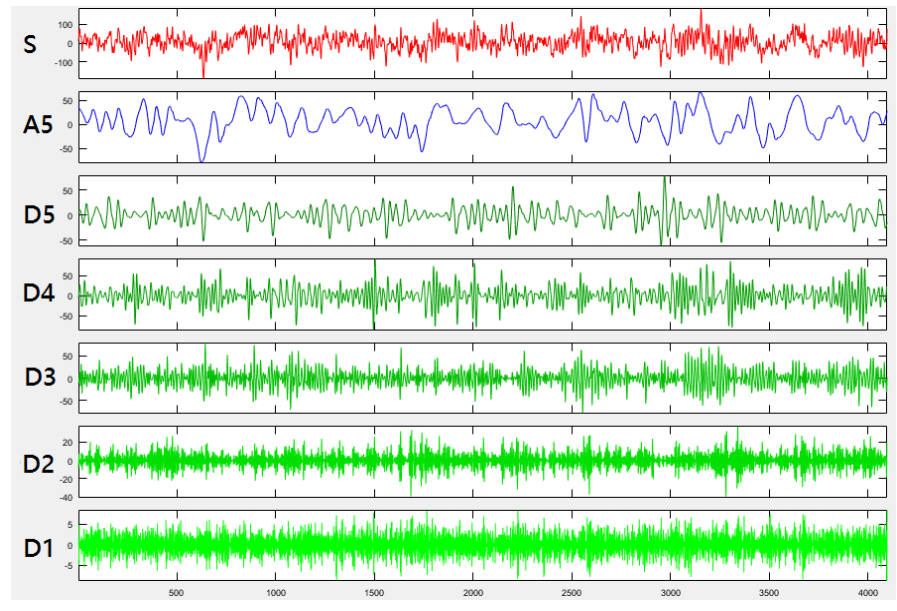

Fig. 3 Decomposition of signal into coefficient. $S$ represent the original signal decompose into the lowest frequency signal (A5) to the highest frequency signal (D1).

\section{Complexity Measurement}

Approximate entropy, Lempel-Ziv complexity, Hurst exponent, Kolmogorov complexity, Shannon entropy and sample entropy are implemented to extract feature in this study. These complexity measurements have difference way in extracting complexity feature but have the same motivation to discriminate normal EEG from interictal and ictal EEG. The complexity measurements are implemented in MatLab 2015.

\section{Approximate Entropy}

Approximate Entropy (ApEn) first was developed by Pincus to measure system complexity (Pincus 1991). In signal analysis, ApEn helped to measure the regularity and predictability of a signal. The implemenation of ApEn in this study is determined by the following procedure.

1) Let a data sequence containing $n$ data points be $S_{n}=\{u(1), u(2)$, $\mathrm{u}(3), \ldots, \mathrm{u}(\mathrm{n})]$

2) Choose value of $m$ and $r$ where $m=$ pattern length and $r=$ criterion of similarity $r=k \times S D$ for $\mathrm{k}=0,0.1,0.2,0.3, \ldots, 0.9$. SD will be present as standard deviation of data $S_{n}$

3) Let $X$ be sequence of $x(i)$ such that $x(i)=[u(i), u(i+1), u(i+2), \ldots, u(i+m-1)]$ where $i=1,2,3, \ldots,(n-m+1)$

4) Find the distance between vector $\mathrm{x}(\mathrm{i})$ and $\mathrm{x}(\mathrm{j})$ by using formula $d\left[x, x^{*}\right]=\max _{a}\left|u(a)-u^{*}(a)\right|$, if $d\left[x, x^{*}\right]<r$ the pattern are likely similar

5) Calculate $C_{i}^{m}=\frac{\text { number of } d\left[x, x^{*}\right] \text { less than } r}{(n-m+1)}$ and $C_{i}^{m+1}=$ $\underline{\text { number of } d\left[x, x^{*}\right] \text { less than } r}$

6) Define $\quad \Phi^{m}(r)=\frac{\sum_{i=1}^{n-m+1} \ln \left(C_{i}^{m}(r)\right)}{n-m+1}$ and $\Phi^{m+1}(r)=$ $\frac{\sum_{i=1}^{n-m+1} \ln \left(C_{i}^{m}(r)\right)}{n-m+1}$

7) $\operatorname{ApEn}(m, r, n)$ is determined as follow: $\operatorname{ApEn}(m, r, n)=$ $\Phi^{m}(r)-\Phi^{m+1}(r)$

Large ApEn value indicates the signal is unpredictable and irregular while a small ApEn value indicate higher regularity and repetitive pattern. To determine the ApEn, the (m) and (r) are set to 2 and $0.2 x S D$ respectively based on Srinivasan et. al (Srinivasan et al. 2007) to obtain the highest percentage of efficiency.

\section{Lempel-Ziv Complexity}

To compute LZ complexity, the signal S(n) must be transformed into a symbolic sequence first. The EEG signal was converted into a binary sequence (Aboy et al. 2006) by comparing with threshold Td as shown in (2) (3) .

$$
S=s(1), s(2), \ldots, s(n)
$$

where

$$
s(i)=\left\{1, \text { if } s(i)<T_{d} 0\right. \text {, otherwise }
$$

Commonly the median is used as the threshold due to its robustness to outliers (Aboy et al. 2006). The signal S is scanned from left to right and the complexity counter $\mathrm{c}(\mathrm{n})$ will increase each time new subsequence of consecutive character is come up. The complexity value are obtained by following algorithm (Abásolo et al. 2007):

1) Let $P$ be a signal which contain two subsequences, $S$ and $Q$. Let SQ be the sequence of S and Q, while SQ $\pi$ is derived from SQ after its last character deleted. Let $\mathrm{v}(\mathrm{SQ} \pi)$ indicate the vocabulary of all different subsequences of $\mathrm{SQ} \pi$. For started, value $\mathrm{c}(\mathrm{n})=1$, $\mathrm{S}=\mathrm{s}(1), \mathrm{Q}=\mathrm{s}(2)$ and $\mathrm{SQ} \pi=\mathrm{s}(1)$.

2) For generalization, $\mathrm{S}=\mathrm{s}(1), \mathrm{s}(2), \ldots, \mathrm{s}(\mathrm{r}), \mathrm{Q}=\mathrm{s}(\mathrm{r}+1)$ and $\mathrm{SQ} \pi=\mathrm{s}(1)$, $\mathrm{s}(2), \ldots, \mathrm{s}(\mathrm{r})$. If $\mathrm{Q}$ fits in $\mathrm{v}(\mathrm{SQ} \pi)$, then $\mathrm{Q}$ is a subsequence of $\mathrm{SQ} \pi$ which is not a new sequence.

3) Renew $\mathrm{Q}$ to be $\mathrm{s}(\mathrm{r}+1)$. Value $\mathrm{Q}$ is checked whether it belongs to $\mathrm{v}(\mathrm{SQ} \pi)$ or not.

4) Step 3 is repeat until Q does not belongs to $v(S Q \pi)$, which means $\mathrm{Q}$ is not a subsequence of $\mathrm{v}(\mathrm{SQ} \pi)$.

5) $\mathrm{SQ} \pi$ is a new sequence, so value of $\mathrm{c}(\mathrm{n})$ increase by one.

6) $\mathrm{S}$ is renewed to be $\mathrm{S}=\mathrm{s}(1), \mathrm{s}(2), \ldots, \mathrm{s}(\mathrm{r}+\mathrm{i})$ and $\mathrm{Q}=\mathrm{s}(\mathrm{r}+\mathrm{i}+1)$.

7) Repeat procedure until $Q$ is the last character.

The complexity value depends on number of different subsequences in P. To acquire complexity independent to sequence length, $c(n)$ need to be normalized as follow (4):

$$
C(n)=\frac{c(n) \times \log _{3} n}{n}
$$

The value of $\mathrm{C}(\mathrm{n})$ obtained are used as feature in this study to measure the complexity of the EEG

\section{Hurst Exponent}

Hurst exponent (HE) was introduced by Harold Edward Hurst to determine dam sizing for the Nile River's based on observation of rain and drought condition for a long period of time (Hurst 1956). The concept is to measure the presence or absence of long-range dependence and its extent in a time series. HE can be defined as (5)

$$
H=\frac{\log \frac{R}{S}}{\log T}
$$

Where $R / S$ is the corresponding value of rescaled range and $\mathrm{T}$ is the duration of the sample of data. Hurst exponent values range from 0 to 1. Supposing that the value of exponent is less than 0.5, the time series data are likely to be anti-correlated, whereby an increase will be followed by decrease and a decrease will be followed increase value. Exponent value of 0.5 indicates that the time series it is totally random and unpredictable. Exponent values greater than 0.5 are claim to be correlated whereby an increase in the values will likely to be followed by increase value and decreasing value will likely to be followed by decreasing value. Application of HE in automated epileptic discharge detection has been conducted by Hosseini et al, Geng et. al and Kishky et al. It is proven that the exponent values of interictal EEG are higher than ictal EEG by Geng et al (Geng et al. 2011) and Kishky (El-Kishky 2012). The implementation of $\mathrm{HE}$ in this study is begin with dispersional analysis or also known as Aggregated Variance method (Murad S. Taqqu, Vadim Teverovsky 1995) on the EEG segment. Matlab polyfit is applied after the analysis to estimate the HE of the time series.

\section{Kolmogorov Complexity}

Kolmogorov Complexity (KC) was developed by Andrey Kolmogorov to defined as the length of the shortest possible description of a time series (Kolmogorov 1963). The Kolmogorov Complexity is approximate the entropy of the source distribution for the process generating the sequence. The difference from entropy is it relates to the 
specific string being considered rather than the source distribution (Evans et al. 2002). KC can be defined as equation (6)

$$
K_{\varphi}(x)=\left\{\min _{\varphi(p)=x} l(p)\right\}
$$

Where $\varphi$ represents a universal computer, $\mathrm{p}$ represents a program and $\mathrm{x}$ represent a string. Recent study has presented its application in detecting epileptic discharge (Pratiher et al. 2016). The result shows increasing value for ictal epileptic discharge activity compare to normal and interictal epileptic discharge. Random signal rather has high Kolmogorov Complexity value (Evans et al. 2002)

\section{Shannon Entropy}

Claude Shannon introduced Shannon entropy (SE) in Information Theory (Shannon 1948). It computes the degree of uncertainty that exist in a system. SE can be defined as equation (7)

$$
H(X)=-\sum_{i=0}^{N-1} p\left(x_{i}\right) \log _{2} p\left(x_{i}\right)
$$

Which yield the value of the complexity in $x_{i}$ and $p$ stand for probability of pattern. Recent publication regarding Shannon entropy has shown good performance in detecting seizure (Alsharabi et al. 2016).

\section{Sample Entropy}

Sample entropy (SampEn) is another popular complexity measurement technique developed by Richman and Moorman (Richman \& Moorman, 2000) as a modification of ApEn. Compared to ApEn, this technique has advantages in application on short time series data and consistency. SampEn is a negative probability algorithm where two sequences similar for $m$ points will stay the same at the next point. This is due to self-matches are not included in computing the probability. To compute the SampEn, the pattern length (m), number of data point $(\mathrm{N})$ and vector comparison distance (r) must be declared. Since the time series length is finite, the SampEn can be defined as follow (8)

$$
\operatorname{SampEn}(m, r, N)=\ln \left[\frac{B^{m}(r)}{A^{m}(r)}\right]
$$

Value $r$ in this study is fixed to 0.2 times the standard deviation of time series and $m$ is set to two to enable fair comparison with ApEn. Similar to ApEn, larger values of SampEn indicate to more complexity in the data and vice versa. SampEn value decrease when epileptic discharge is presence in EEG (Song 2010). Implementation SampEn algorithm in MatLab is not much difference compare to ApEn procedure mentioned before, only in usage of Chebyshev to find distance between vector to exclude self matching case.

\section{Classification}

Support vector machine (SVM) is implemented as classification in this study due to its excellence ability in handling high dimensionality feature vector, flexibility in modelling diverse sources of data and high accuracy (Ben-hur \& Weston 2010). SVM function by constructing a hyperplane with the largest margin to separate between two classes. Employment of radial basis function (RBF) with sigma of 1 as SVM kernel has advantage which it is the best in handling relation between attributes and class labels that are non-linear (Hsu et al. 2008). The SVM is implemented using MATLAB library function. 50 data were randomly selected as train data from each dataset. The remaining of the data are used as classifier data.

\section{RESULTS AND DISCUSSION}

\section{Signal Complexity without MRA}

Initially, we computed the average complexity of the signal using each technique without the MRA. Table III demonstrate the complexity value of the methods for dataset $\mathrm{A}, \mathrm{B}$ and $\mathrm{C}$.
Table 3 Complexity value of ApEn, LZ, HE, SE, KC and SampEn for each dataset.

\begin{tabular}{cccc}
\hline $\begin{array}{c}\text { Complexity } \\
\text { Measurement }\end{array}$ & A & $\begin{array}{c}\text { Dataset } \\
\text { B }\end{array}$ & C \\
\hline ApEn & 0.997 & 0.614 & 0.493 \\
LZ & 0.069 & 0.066 & 0.023 \\
HE & 0.657 & 0.603 & 0.435 \\
SE & 2.373 & 2.460 & 5.511 \\
KC & 0.544 & 0.344 & 0.383 \\
SampEn & 1.011 & 0.618 & 0.496 \\
\hline
\end{tabular}

In Table 3, we observe the decreasing of complexity value in ApEn, LZ, HE and SampEn. Decrease of complexity value is due to presence epileptic discharge in the EEG signal. KC shows slight increase in Set C compare to Set B. However, SE tends to behave in the opposite manner and this finding is correlate with the study of brain complexity due to anaesthesia (Ferenets et al.,. 2006). HE characterize the Set A and $\mathrm{B}$ as correlated signal while the Set $\mathrm{C}$ is anti-correlated signal. Fig. 4 support the trends of the complexity value between the three datasets.

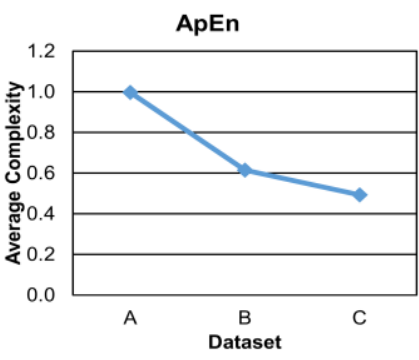

(a)

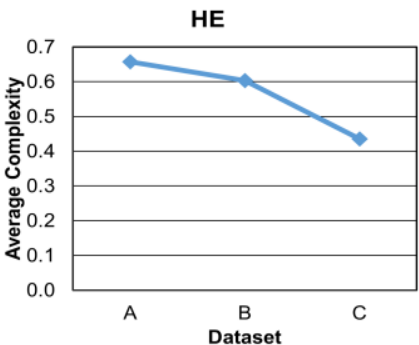

(c)

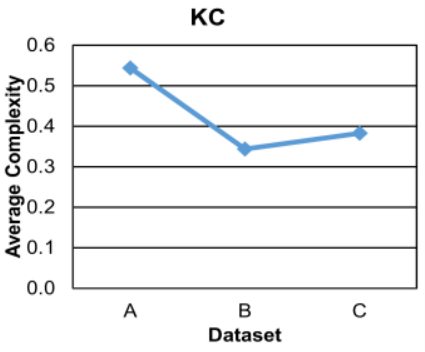

(e)

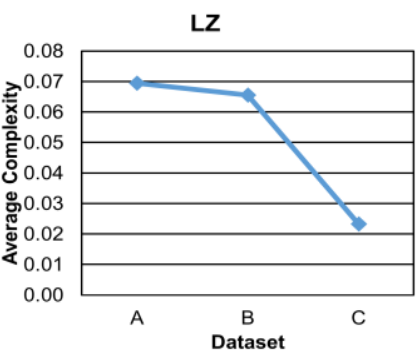

(b)

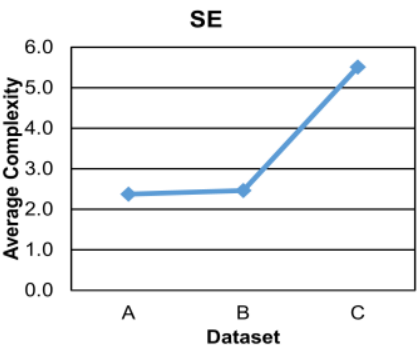

(d)

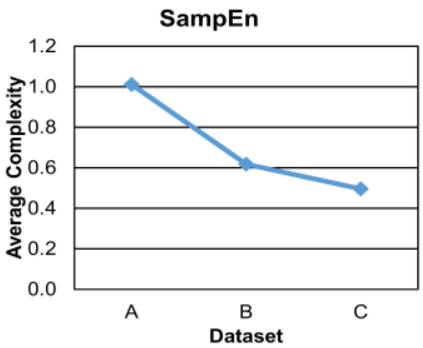

(f)
Fig. 4 Graph trends of complexity value of each data set for (a) ApEn (b) LZ (c) HE (d) SE (e) KC (f) SampEn.

\section{Signal Complexity with MRA}

Next we implemented the MRA on the complexity measurement. The signal is decomposed into six sub-bands frequency. The complexity can be assessed in each sub-bands and the average complexity value is tabulated in Table 4. 
Table 4 Average sub-band complexity value of ApEn, LZ, HE, SE, KC and SampEn for each dataset.

\begin{tabular}{|c|c|c|c|c|c|c|c|c|c|c|c|c|c|c|c|c|c|c|}
\hline & \multicolumn{3}{|c|}{ ApEn } & \multicolumn{3}{|c|}{ LZ } & \multicolumn{3}{|c|}{ HE } & \multicolumn{3}{|c|}{ SE } & \multicolumn{3}{|c|}{ KC } & \multicolumn{3}{|c|}{ SampEn } \\
\hline & A & B & C & A & B & C & A & B & C & A & B & C & A & B & C & A & B & C \\
\hline A & 0.23 & 0.27 & 0.23 & 0.23 & 0.27 & 0.32 & 0.75 & 0.68 & 0.65 & 1.39 & 1.86 & 2.83 & 0.10 & 0.12 & 0.14 & 0.16 & 0.19 & 0.23 \\
\hline 5 & 9 & 6 & 1 & 9 & 6 & 0 & 5 & 1 & 4 & 9 & 6 & 5 & 9 & 5 & 2 & 2 & 1 & 2 \\
\hline D & 0.66 & 0.64 & 0.47 & 0.66 & 0.64 & 0.65 & 0.24 & 0.24 & 0.25 & 0.84 & 1.30 & 3.94 & 0.24 & 0.23 & 0.23 & 0.43 & 0.40 & 0.47 \\
\hline 5 & 4 & 7 & 2 & 4 & 7 & 4 & 8 & 8 & 8 & 0 & 0 & 6 & 4 & 8 & 6 & 6 & 9 & 4 \\
\hline D & 1.16 & 1.10 & 0.56 & 1.16 & 1.10 & 1.07 & 0.05 & 0.05 & 0.05 & 1.09 & 1.20 & 4.29 & 0.37 & 0.35 & 0.35 & 0.59 & 0.54 & 0.56 \\
\hline 4 & 9 & 2 & 5 & 9 & 2 & 1 & 0 & 1 & 5 & 8 & 2 & 7 & 5 & 8 & 4 & 8 & 7 & 8 \\
\hline D & 1.76 & 1.68 & 0.62 & 1.76 & 1.68 & 1.62 & 0.15 & 0.16 & 0.15 & 1.19 & 0.65 & 3.92 & 0.51 & 0.50 & 0.48 & 0.81 & 0.70 & 0.63 \\
\hline 3 & 3 & 4 & 6 & 3 & 4 & 6 & 9 & 2 & 0 & 0 & 4 & 9 & 5 & 1 & 6 & 0 & 9 & 2 \\
\hline D & 2.11 & 2.26 & 0.59 & 2.11 & 2.26 & 1.99 & 0.05 & 0.05 & 0.07 & 0.85 & 0.27 & 2.34 & 0.61 & 0.63 & 0.59 & 1.02 & 0.92 & 0.60 \\
\hline 2 & 6 & 7 & 8 & 6 & 7 & 6 & 6 & 6 & 5 & 9 & 2 & 1 & 1 & 6 & 1 & 4 & 9 & 5 \\
\hline D & 3.20 & 3.27 & 0.76 & 3.20 & 3.27 & 3.19 & 0.07 & 0.12 & 0.14 & 0.02 & 0.00 & 0.53 & 0.79 & 0.80 & 0.79 & 1.57 & 1.45 & 0.77 \\
\hline 1 & 9 & 9 & 8 & 9 & 9 & 8 & 0 & 9 & 2 & 4 & 6 & 4 & 5 & 9 & 1 & 3 & 3 & 6 \\
\hline
\end{tabular}

Graph of average complexity for all complexity measurements are plotted in Fig. 5. A rising pattern of complexity value can be observed in ApEn, LZ, KC and SampEn. These techniques exhibit close complexity value between Set A, B and C in lower sub-band frequency and the value are spreading at the higher frequency. On the other hand, SE shows a declining trend as the frequency increase. A huge gap can be observed between Set $\mathrm{C}$ with Set A and B, indicating the SE can distinguish seizure from normal and interictal very well. HE shows a random trend where it starts with steep decline in the lower frequency bands follows by fluctuation in higher frequency bands. Implementation of MRA with HE describes all the dataset as highly anti-correlated. This result contradicts to HE without MRA.

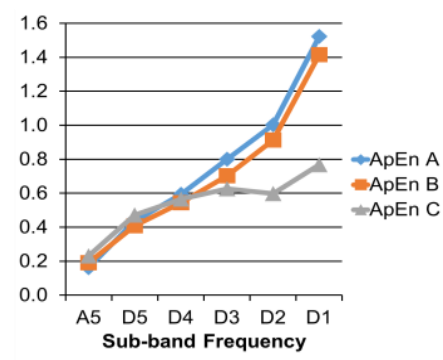

(a)

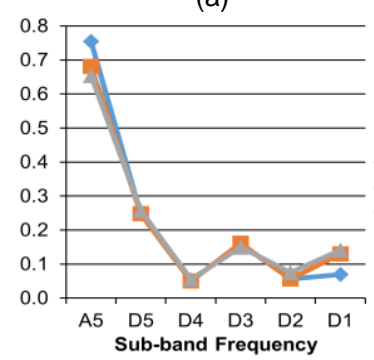

(c)

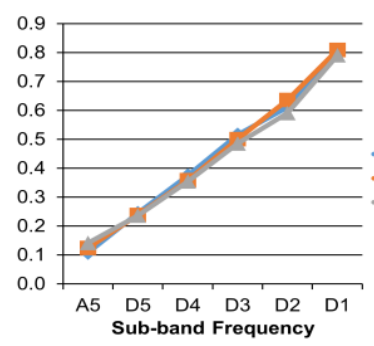

(e)

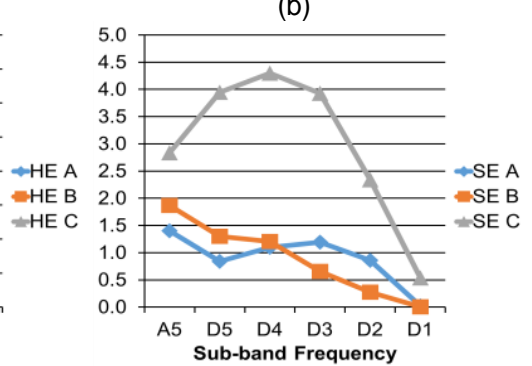

(d)

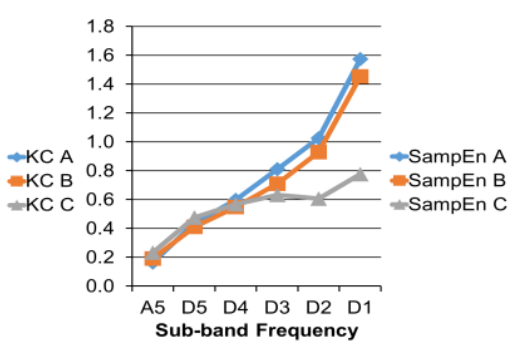

(f)
Fig. 5 Sub-band complexity value of each data set for (a) ApEn (b) LZ (c) HE (d) SE (e) KC (f) SampEn

\section{Evaluation of performance with and without MRA}

We compare the performance of the complexity measurement with and without MRA in terms of accuracy, sensitivity and specificity. The result is tabulated in Table V. MRA manage to increase the performance in some of the complexity measurement. In overall, ApEn and SE are the best techniques to be implemented with MRA as it will improve the accuracy, sensitivity and specificity in differentiating normal with interictal (Set A and B), normal with ictal (Set A and C) also interictal with ictal (Set B and C). SE is better than ApEn based on the higher accuracy in classification of Set A with B and Set B with C. However, ApEn has advantage as it takes into account the temporal order of points in a time sequence thus making it more preferred measure of complexity (Ocak 2009). Disadvantage of ApEn is it unsuitable to be applied for short time series data and it appears that SampEn is fit better for this task (Yentes et al. 2013). Other complexity measure shows a trade off in distinguishing between two groups when MRA is applied i.e. KC and SampEn exhibits lower accuracy in distinguishing Set $\mathrm{A}$ and $\mathrm{B}$ while improving the detection rate in between Set A and C and Set B and C. MRA affect LZ and HE the worst since it deteriorates the performance in differentiating Set $\mathrm{A}$ and $\mathrm{C}$ and Set B and C.

Other effect of MRA is it tends to increase the computational time due to the increase of number of sub-band frequency or feature. The sub-band frequency is directly proportional to the number of level of decomposition, thus it is recommended to select the optimum number based on research requirement. A method of using sparseness measurement to select suitable level of decomposition is proposed (Lei et al. 2013). Simpler method in choosing level of decomposition is based on frequency range needed to be analysed (Deshprabhu \& Shenvi 2015). Level of decomposition can be selected based on classification to physiological band i.e. Delta, Theta, Alpha, Beta, Gamma (Shen et al. 2013).

Fig. 6 represents the average time taken to compute an EEG signal with duration of 23.3 seconds and sampling rate of $173.61 \mathrm{~Hz}$. This study is conducted using computer with specification: Intel Pentium G3250 3.2Ghz, 8GB RAM on Window 7 operational system. A pattern can be observed that good method tends to have longer computational time. Longer computational time requires when processing using SE while HE has the shortest computational time. Computational time is crucial when developing epileptic discharge algorithm especially in intensive care environment. Based on Fig. 6, SE takes 2.9 seconds to process a signal. When MRA with 6 sub-band apply on the SE, approximately it will take 17.4 seconds to finish. It is inefficient to deploy this algorithm in Intensive Care Unit (ICU) with such duration. Thus, it is recommended to use more sophisticated computer or switching to another complexity measure with tolerable performance. Although algorithm such as LZ and $\mathrm{KC}$ take less computational time, it should be noted both need extra process to change the signal into binary sequence. 
Table V Comparison of performance based on percentage (\%) of accuracy (Acc), sensitivity (Sens) and specificity (Spec) on non-MRA and MRA method. Asterisk (*) represent highest accuracy achieved.

\begin{tabular}{|c|c|c|c|c|c|c|c|c|c|c|c|c|c|c|c|c|c|c|}
\hline \multirow[b]{2}{*}{$\begin{array}{l}\text { Dataset } \\
\text { Metric }\end{array}$} & \multicolumn{9}{|c|}{$\begin{array}{l}\text { Non-MRA } \\
\text { AC }\end{array}$} & \multicolumn{9}{|c|}{ MRA } \\
\hline & Acc & $\begin{array}{r}A B \\
\text { Sens } \\
\end{array}$ & Spec & Acc & $\begin{array}{c}\text { AC } \\
\text { Sens }\end{array}$ & Spec & Acc & $\begin{array}{r}B C \\
\text { Sens } \\
\end{array}$ & Spec & Acc & $\begin{array}{c}A B \\
\text { Sens }\end{array}$ & Spec & Acc & $\begin{array}{c}\text { AC } \\
\text { Sens }\end{array}$ & Spec & Acc & $\begin{array}{c}B C \\
\text { Sens }\end{array}$ & Spec \\
\hline ApEn & 74 & 75 & 92.9 & 98 & 96.2 & 100 & 88 & 95.2 & 82.8 & $89^{*}$ & 91.5 & 86.8 & $100^{*}$ & 100 & 100 & $94^{*}$ & 95.8 & 92.3 \\
\hline LZ & 49 & 48 & 49.3 & $91^{*}$ & 95.6 & 87.3 & $89^{*}$ & 95.3 & 84.2 & $83^{*}$ & 80 & 86.7 & 86 & 90.9 & 82.1 & 88 & 88 & 88 \\
\hline HE & 65 & 60.9 & 74.2 & $89^{*}$ & 83.1 & 97.6 & $80^{*}$ & 73.4 & 91.7 & $70^{*}$ & 71.7 & 68.5 & 78 & 75.9 & 80.4 & 54 & 53.4 & 54.8 \\
\hline KC & $95^{*}$ & 90.9 & 100 & 92 & 86.2 & 100 & 52 & 51.1 & 58.3 & 84 & 85.4 & 82.7 & $95^{*}$ & 95.9 & 94.1 & $89^{*}$ & 86.8 & 91.5 \\
\hline SE & 69 & 63.8 & 80.6 & 100 & 100 & 100 & 96 & 98 & 94.2 & $98^{*}$ & 96.2 & 100 & $100^{*}$ & 100 & 100 & $97^{\star}$ & 100 & 94.3 \\
\hline SampEn & $92^{*}$ & 90.4 & 93.8 & 98 & 96.2 & 100 & 59 & 58.8 & 59.2 & 88 & 89.6 & 86.5 & $100^{*}$ & 100 & 100 & $94^{*}$ & 95.8 & 92.3 \\
\hline
\end{tabular}

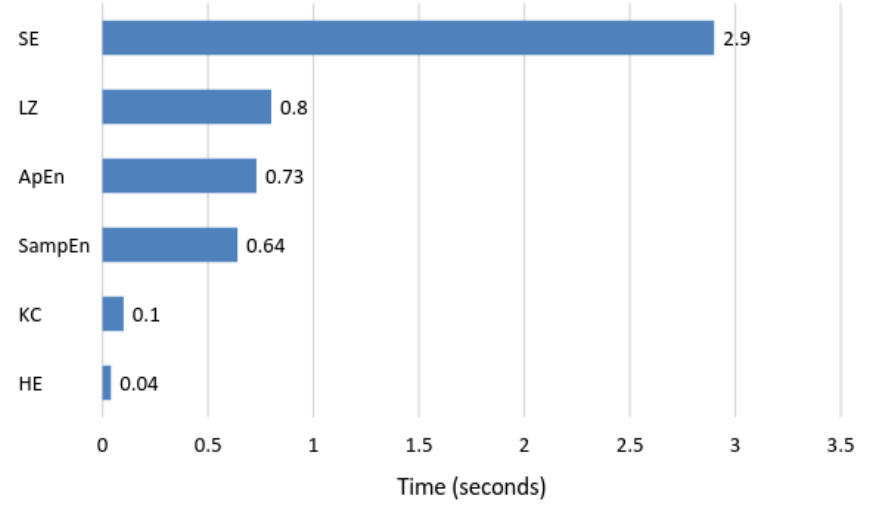

Fig. 6 Computational time of complexity technique in descending order of time.

We have encounter difficulty in comparing different complexity measure of ApEn and SampEn due parameter values selection. The parameters were chosen according to the literature based on the similar application. Pattern length is fix to $m=2$ for ApEn (Srinivasan et al. 2007) and SampEn (Yentes et al. 2013). For the criterion of similarity, $r$, there are varies of parameter value claims to be the best such that $r=$ $g x$ standard deviation of data where $g=0.1,0.2, \ldots, 0.5$ (Jiang et al. 2015; Song \& Zhang 2013). Value of $g=0.2$ is decided for ApEn and SampEn to enable fair comparison although there might be possibility one measure performs better than another when using different value.

\section{CONCLUSION}

In this study, we have demonstrated the effect of MRA on complexity measurement of epileptic discharge shows improvement in detection, but it increases the computational time. ApEn and SE are the two most outstanding variables while $\mathrm{HE}$ and $\mathrm{LZ}$ is not suitable to be used in MRA implementation. Trade-off between performance and computational time must be balanced to obtain excellent epileptic discharge detection algorithm. Our finding showed ApEn has the best balance between performance and computational time when used with MRA. This combination is expected to be beneficial for neurologist in reducing diagnosing time for epilepsy patient.

\section{ACKNOWLEDGEMENT}

This work was financially supported by the Universiti Kebangsaan Malaysia under LRGS/2013/UMK-UKM/SS/04

\section{REFERENCES}

Abásolo, D., James, C. J., Hornero, R. 2007. Non-linear analysis of intracranial electroencephalogram recordings with approximate entropy and Lempel-Ziv complexity for epileptic seizure detection. $29^{\text {th }}$ Annual International Conference of the IEEE Engineering in Medicine and Biology Society. IEEE
Engineering in Medicine and Biology Society. 22-26 August. Lyon, France: IEEE, 1953-1956.

Aboy, M., Hornero, R., Abásolo, D., Álvarez, D. 2006. Interpretation of the Lempel-Ziv complexity measure in the context of biomedical signal analysis. IEEE Transactions on Biomedical Engineering 53(11), 2282-2288.

Adeli, H., Zhou, Z., Dadmehr, N. 2003. Analysis of EEG records in an epileptic patient using wavelet transform. Journal of Neuroscience Methods 123(1), 69-87.

Alotaiby, T. N., Alshebeili, S. A., Alshawi, T., Ahmad, I., Abd El-Samie, F. E. 2014. EEG seizure detection and prediction algorithms: a survey. EURASIP Journal on Advances in Signal Processing 2014(183), 1-21.

Alsharabi, K., Ibrahim, S., Djemal, R., Alsuwailem, A. 2016. A DWT-entropyANN based architecture for epilepsy diagnosis using EEG signals. 2nd International Conference on Advanced Technologies for Signal and Image Processing. 21-23 March. Monastir, Tunisia: IEEE, 288-291.

Andrzejak, R. G., Schindler, K., Rummel, C. 2012. Nonrandomness, nonlinear dependence, and nonstationarity of electroencephalographic recordings from epilepsy patients. Physical Review E - Statistical, Nonlinear, and Soft Matter Physics 86(4).

Ben-hur, A., Weston, J. 2010. A user' s guide to support vector machines preliminaries : Linear classifiers. Methods in Molecular Biology, 609, 223239.

Blanco, S., Garay, A., Coulombie, D. 2013. Comparison of frequency bands using spectral entropy for epileptic seizure prediction. ISRN Neurology 2013, 287327.

Clemson, P., Lancaster, G., Stefanovska, A. 2016. Reconstructing timedependent dynamics. Proceedings of the IEEE 104(2): 223-241.

Deshprabhu, A. A., Shenvi, N. 2015. Sub-band decomposition of EEG signals and feature extraction for epilepsy classification. International Journal of Advanced Research in Computer and Communication Engineering 4(3), 108111.

El-Kishky, A. 2012. Assessing entropy and fractal dimensions as discriminants of seizures in EEG time series. 2012 11th International Conference on Information Science, Signal Processing and their Applications, ISSPA 2012. 2-5 July. Montreal, QC, Canada: IEEE, 92-96.

Evans, S. C., Hershey, J. E., Saulnier, G., Series, T. I. 2002. Kolmogorov Complexity Estimation and Analysis (October). Retrieved from https://pdfs.semanticscholar.org/a323/340daab198794f245135eb07bce22901 2301.pdf

Fathillah, M. S., Jaafar, R., Chellappan, K., Remli, R., Zainal, W. A. W. 2017. Complexity analysis on EEG signal via Lempel-Ziv and approximate entropy: Effect of multiresolution analysis. International Medical Device and Technology Conference 2017. 6-7 September. Johor Bahru, Malaysia.

Ferenets, R., Lipping, T., Anier, A., Jäntti, V., Melto, S., Hovilehto, S. 2006 Comparison of entropy and complexity measures for the assessment of depth of sedation. IEEE Transactions on Biomedical Engineering 53(6), 10671077.

Fisher, R. S., Van Emde Boas, W., Blume, W., Elger, C., Genton, P., Lee, P., Engel, J. 2005. Epileptic seizures and epilepsy: Definitions proposed by the International League Against Epilepsy (ILAE) and the International Bureau for Epilepsy (IBE). Epilepsia 46(4), 470-472.

Gajic, D., Djurovic, Z., Gligorijevic, J., Di Gennaro, S., Savic-Gajic, I. 2015. Detection of epileptiform activity in EEG signals based on time-frequency and non-linear analysis. Frontiers in Computational Neuroscience 9, 1-16.

Geng, S., Zhou, W., Yuan, Q., Cai, D., Zeng, Y. 2011. \{EEG\} non-linear feature extraction using correlation dimension and Hurst exponent. Neurological research 33(9), 908-912.

Grassberger, P., Schreiber, T., Schaffrath, C. 1991. Nonlinear Time Sequence Analysis. International Journal of Bifurcation and Chaos 1(3), 521-547.

Hsu, C.-W., Chang, C.-C., and C.-J. L. 2008. A Practical Guide to Support Vector Classification. BJU international 101(1), 1396-1400. 
Hurst, H. E. 1956. The problem of long-term storage in reservoirs. International Association of Scientific Hydrology. Bulletin 1(3), 13-27.

Jiang, G. J. A., Fan, S.-Z., Abbod, M. F., Huang, H.-H., Lan, J.-Y., Tsai, F.-F., Chang, H.-C., et al. 2015. Sample entropy analysis of EEG signals via artificial neural networks to model patients' consciousness level based on anesthesiologists experience. BioMed Research International 2015, 1-8.

Klonowski, W. 2009. Everything you wanted to ask about EEG but were afraid to get the right answer. Nonlinear Biomedical Physics 3, 2.

Kolmogorov, A. N. 1963. On tables of random numbers. Source: Sankhyā: The Indian Journal of Statistics, Series A 25(4), 369-376.

Kumar, Y., Dewal, M. L., Anand, R. S. 2014. Epileptic seizure detection using DWT based fuzzy approximate entropy and support vector machine. Neurocomputing 133, 271-279.

Lei, L., Wang, C. \& Liu, X. 2013. Discrete wavelet transform decomposition level determination exploiting sparseness measurement. International Journal of Electrical, Computer, Energetic, Electronic and Communication Engineering 7(9), 691-694.

Li, X., Hu, B., Sun, S., Cai, H. 2016. EEG-based mild depressive detection using feature selection methods and classifiers. Computer Methods and Programs in Biomedicine 136, 151-161.

Murad S. Taqqu, Vadim Teverovsky, W. W. 1995. Estimators for long-range dependence: An empirical study. Fractals 3(4), 785-798.

Nagaraj, V., Lee, S. T., Krook-magnuson, E., Soltesz, I., Benquet, P., Irazoqui, P. P., Netoff, T. I. 2015. Future of Seizure Prediction and Intervention: Closing the Loop. Journal of Clinical Neurophysiology: Official publication of the American Electroencephalographic Society 32, 194-206.

Natarajan, K., Acharya U, R., Alias, F., Tiboleng, T., Puthusserypady, S. K. 2004. Nonlinear analysis of EEG signals at different mental states. Biomedical Engineering Online 3(1), 7, 1-11.

Ocak, H. 2009. Automatic detection of epileptic seizures in EEG using discrete wavelet transform and approximate entropy. Expert Systems with Applications 36(2 PART 1), 2027-2036.

Omerhodzic, I., Avdakovic, S., Nuhanovic, A., Dizdarevic, K. 2010. Energy distribution of EEG Signals : EEG signal wavelet-neural network classifier. Neurocomputing 2(1), 210-215.

Pincus, S. M. 1991. Approximate entropy as a measure of system complexity. Proceedings of the National Academy of Sciences 88(6), 2297-2301.

Pratiher, S., Patra, S., Bhattacharya, P. 2016. On the marriage of Kolmogorov complexity and multi-fractal parameters for epileptic seizure classification.
Proceedings of the 2016 2nd International Conference on Contemporary Computing and Informatics. 4-17 December. Noida, India: IEEE, 831-836.

Richman, J. S., Moorman, J. R. 2000. Physiological time-series analysis using approximate entropy and sample entropy. American Journal of PhysiologyHeart and Circulatory Physiology 278, H2039-H2049.

Shannon, C. E. 1948. A mathematical theory of communication. The Bell System Technical Journal 27, 379-423.

Shen, C.-P., Chen, C.-C., Hsieh, S.-L., Chen, W.-H., Chen, J.-M., Chen, C.-M., Lai, F., et al. 2013. High-performance seizure detection system using a wavelet-approximate entropy-fSVM cascade with clinical validation. Clinical EEG and neuroscience 44(4), 247-56.

Smith, S. J. M. 2005. EEG in the diagnosis, classification, and management of patients with epilepsy. Journal of Neurology, Neurosurgery \& Psychiatry 76, ii2-ii7.

Song, Y. 2010. A new approach for epileptic seizure detection: Sample entropy based feature extraction and extreme learning machine. Journal of Biomedical Science and Engineering 3, 556-567.

Song, Y., Zhang, J. 2013. Automatic recognition of epileptic EEG patterns via Extreme Learning Machine and multiresolution feature extraction. Expert Systems with Applications 40(14), 5477-5489.

Srinivasan, V., Eswaran, C., Sriraam, N. 2007. Approximate entropy-based epileptic EEG detection using artificial neural networks. IEEE Transactions on Information Technology in Biomedicine 11(3), 288-295.

Staley, K. J., Dudek, F. E. 2006. Interictal Spikes and Epileptogenesis. Epilepsy Currents 6(6), 199-202.

Stam, C. J. 2005. Nonlinear dynamical analysis of EEG and MEG: Review of an emerging field. Clinical Neurophysiology 116(10), 2266-2301.

Wang, L., Xue, W., Li, Y., Luo, M., Huang, J., Cui, W., Huang, C. 2017. Automatic epileptic seizure detection in EEG signals using multi-domain feature extraction and nonlinear analysis. Entropy 19(6), 1-17.

Yentes, J. M., Hunt, N., Schmid, K. K., Kaipust, J. P., McGrath, D., Stergiou, N. 2013. The appropriate use of approximate entropy and sample entropy with short data sets. Annals of Biomedical Engineering 41(2), 349-365.

Zhang, X. S., Roy, R. J., Jensen, E. W. 2001. EEG complexity as a measure of depth of anesthesia for patients. IEEE Transactions on Biomedical Engineering 48(12), 1424-1433. 